\title{
Self-organization in irradiated semiconductor crystals caused by thermal annealing
}

\author{
M. Zavada ${ }^{1}$, O. Konoreva ${ }^{1}$, P. Lytovchenko ${ }^{1}$, V. Opilat ${ }^{2}$, M. Pinkovska ${ }^{1}$, O. Radkevych ${ }^{3}$, V. Tartachnyk ${ }^{1}$ \\ ${ }^{1}$ Institute for Nuclear Research, NAS of Ukraine, 47, prospect Nauky, 03028 Kyiv, Ukraine \\ ${ }^{2}$ Taras Shevchenko National University of Kyiv, 64/13, Volodymyrska Str., 01601 Kyiv, Ukraine \\ ${ }^{3}$ SE 'SRI of Microdevices' STC 'Institute for Single Cristals', NAS of Ukraine, \\ 3, Pivnichno-Syretska str., 04136 Kyiv, Ukraine \\ Corresponding author: phone +38(044)-525-37-49; e-mail: okskon@meta.ua
}

\begin{abstract}
Annealing of complex semiconductors $\mathrm{GaP}$ and $\mathrm{CdP}_{2}$, irradiated at room temperature by high fluences of electrons within $1 \ldots 30 \mathrm{MeV}$ energy interval and $80 \mathrm{MeV}$ $\alpha$-particles, was carried out, and main electrical parameters (conductivity $\sigma$, carrier concentration $n$ and mobility $\mu$ ) as well as the positron lifetime $\tau$ were studied and analyzed. When the point defect concentration excesses some critical value, defects of new kind are formed: oscillation peaks in the isochronous annealing curve appear, and defects with a high cross-section of defect scattering and capture are created. High temperature annealing of the irradiated sample with increased vacancy concentration causes appearance of the vacancy voids with a lower electron density.
\end{abstract}

Keywords: gallium phosphate, defects, electron irradiation, self-organization, annealing.

doi: https://doi.org/10.15407/spqeo21.02.130

PACS 29.40.-n, 85.30.-z, 85.60.Dw

Manuscript received 08.05.18; revised version received 21.06.18; accepted for publication 27.06.18; published online 03.07.18.

\section{Introduction}

Condensed medium under external energy flows appears to be an open system. In order to describe regular behavior of this system and processes of its structural changes, the methods and principles of synergetic are used.

Synergism might be especially inherent to the samples with a high concentration of radiation defects. Stochastic origin of initial defects and fluctuation character of the created structural defects are the reasons for the variety of all observed phenomena in irradiated crystals. The effect of synergism the most obviously occurs after reaching the definitely critical dose and might manifest itself in atypical behavior of the dose dependence of conductivity, oscillation nature of annealing curves and its additional stages as well as in creation of superlattices $[1,2]$.

Some known phenomena, e.g. the Wagner effect, might be attributed to self-organization, when the samples heated to a certain temperature begin to allocate heat, while transition into the more ordered state due to annihilation of radiation defects. Diffusion of lattice defects, stimulated by radiation, and also swelling the constructions of nuclear reactor, which is caused by the vacancy coagulation, are the self-ordering processes, too. And appearance of long-lasting relaxation processes in crystals directly after irradiation and during annealing might be considered as self-organization [3-5].

\section{Experimental}

Complex semiconductors $\mathrm{GaP}$ and $\mathrm{CdP}_{2}$ irradiated at room temperature by high fluences of electrons within 1...30 MeV energy interval and $80-\mathrm{MeV} \alpha$-particles were studied. The samples were annealled after irradiation within the interval of 140 up to $700 \mathrm{~K}$ for $20 \mathrm{~min}$. The electrical parameters (conductivity $\sigma$, carrier concentration $n$ and mobility $\mu$ ) as well as the positron lifetime $\tau$ were analyzed.

\section{Results and discussion}

It was found that recovery processes of the electrical parameters $(\sigma, \mu, n)$ during annealing of $\mathrm{GaP}$ samples irradiated by $4-\mathrm{MeV}$ electrons with $\Phi=10^{17} \mathrm{~cm}^{-2}$ and $12-\mathrm{MeV}$ electrons, $\Phi=5 \cdot 10^{16} \mathrm{~cm}^{-2}$, were substantially different from the annealing of identical samples irradiated by $1-\mathrm{MeV}$ electrons with $\Phi=10^{17} \mathrm{~cm}^{-2}$ (Figs 1-3). Typical picture of annealing for moderate defect concentrations (Fig. 1) consists of three stages $140 \ldots 160{ }^{\circ} \mathrm{C}, 280^{\circ} \mathrm{C}$ and $470{ }^{\circ} \mathrm{C}$, where phosphorous vacancy $V_{P}$ is annealled at the first stage, gallium vacancy $\mathrm{V}_{\mathrm{Ga}}$ - at the second stage and clusters of point defects are annealled within the third stage limits [6]. 


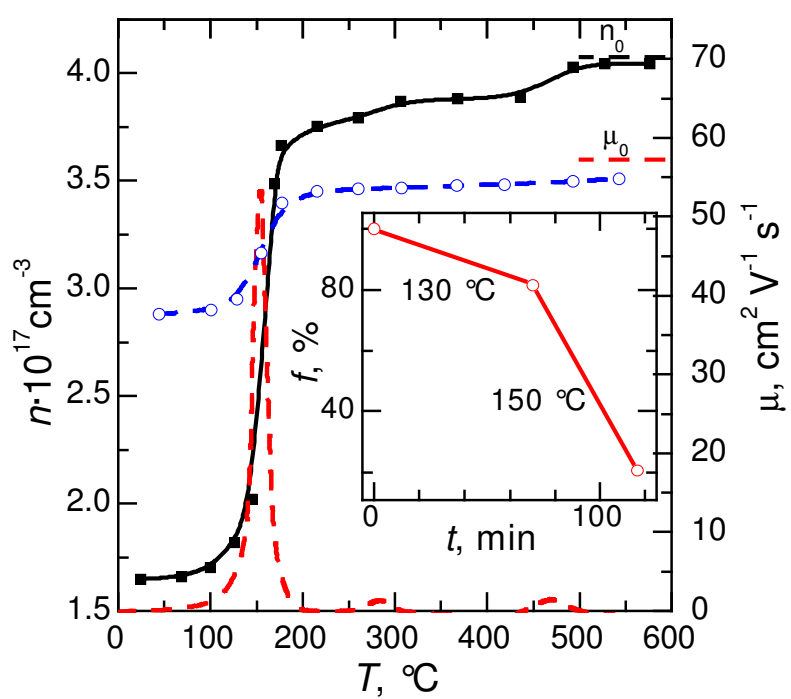

Fig. 1. Recovery of the electron concentration (1) and mobility (2) in $\mathrm{GaP}$ crystal irradiated with $1-\mathrm{MeV}$ electrons, $\Phi=1.2 \cdot 10^{17} \mathrm{~cm}^{-2}$, in the process of isochronous annealing; the first derivative of the concentration with regard to temperature is marked with dots. The results of the isothermal annealing are given in the insert.
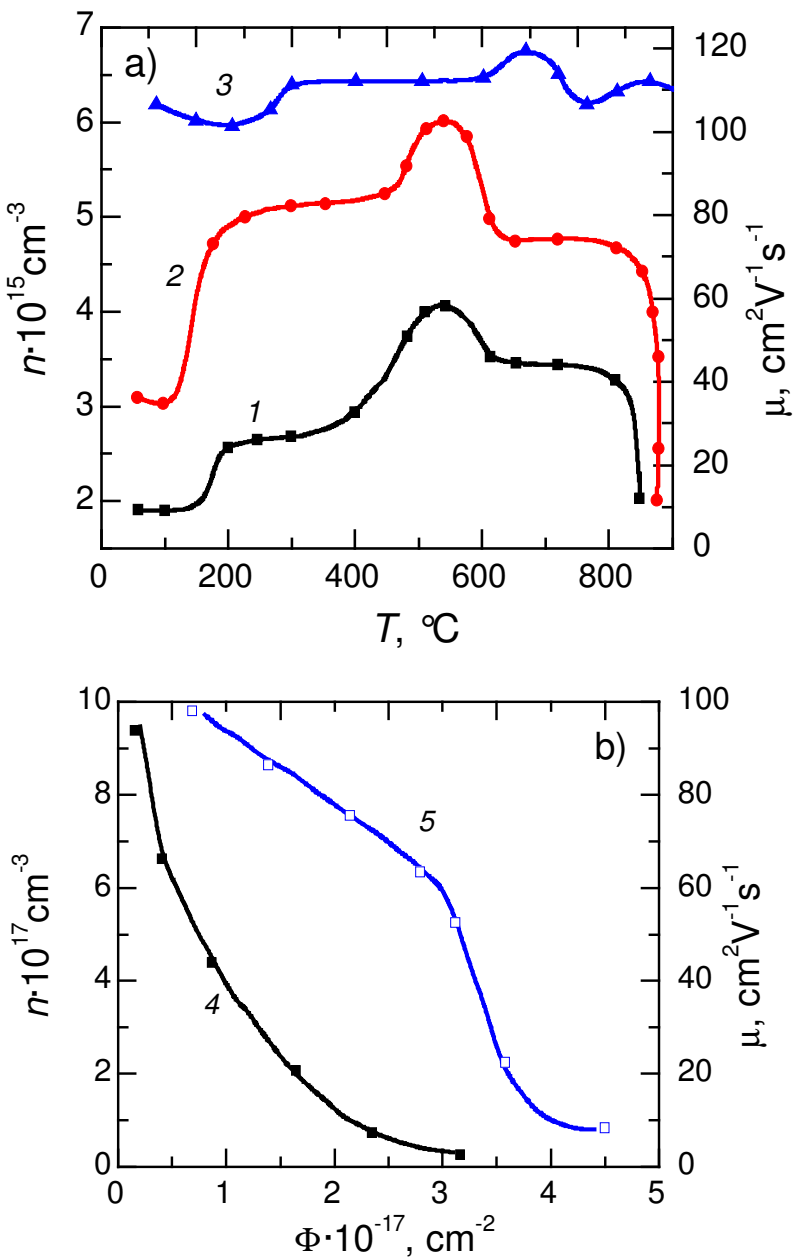

Fig. 2. Recovery of the electrical parameters of GaP crystal irradiated with $4-\mathrm{MeV}$ electrons, $\Phi=10^{17} \mathrm{~cm}^{-2}$, in the process of isochronous annealing; 1 - concentration, 2 - conductivity and 3 - electron mobility on (a). The electron concentration (4) and mobility (5) dependences on the fluence are given on (b).
When the concentration of point defect grows, annealing behavior changes: recovery stage with $500{ }^{\circ} \mathrm{C}$ maximum appears, followed by the negative annealing stage, where the electrical parameters worsened sharply, and the mobility annealing curve has become multistage. This $\mu$ behavior (Fig. 2) might be caused by the appearance of an unstable complex of defects, which affect ambiguously the charge carrier scattering at $400 \ldots 700{ }^{\circ} \mathrm{C}$. Reconstruction of defects is accompanied by their emission.

At high annealing temperatures $\left(T>700^{\circ} \mathrm{C}\right)$, all electron parameters $(\sigma, \mu, n)$ degrade sharply, which is an evidence of formation of the qualitatively another defects with large scattering and capture cross-sections of charge carriers.

The increase of the electron irradiation energy as well as increase of the defect concentration causes new qualitative changes in the annealing process character.
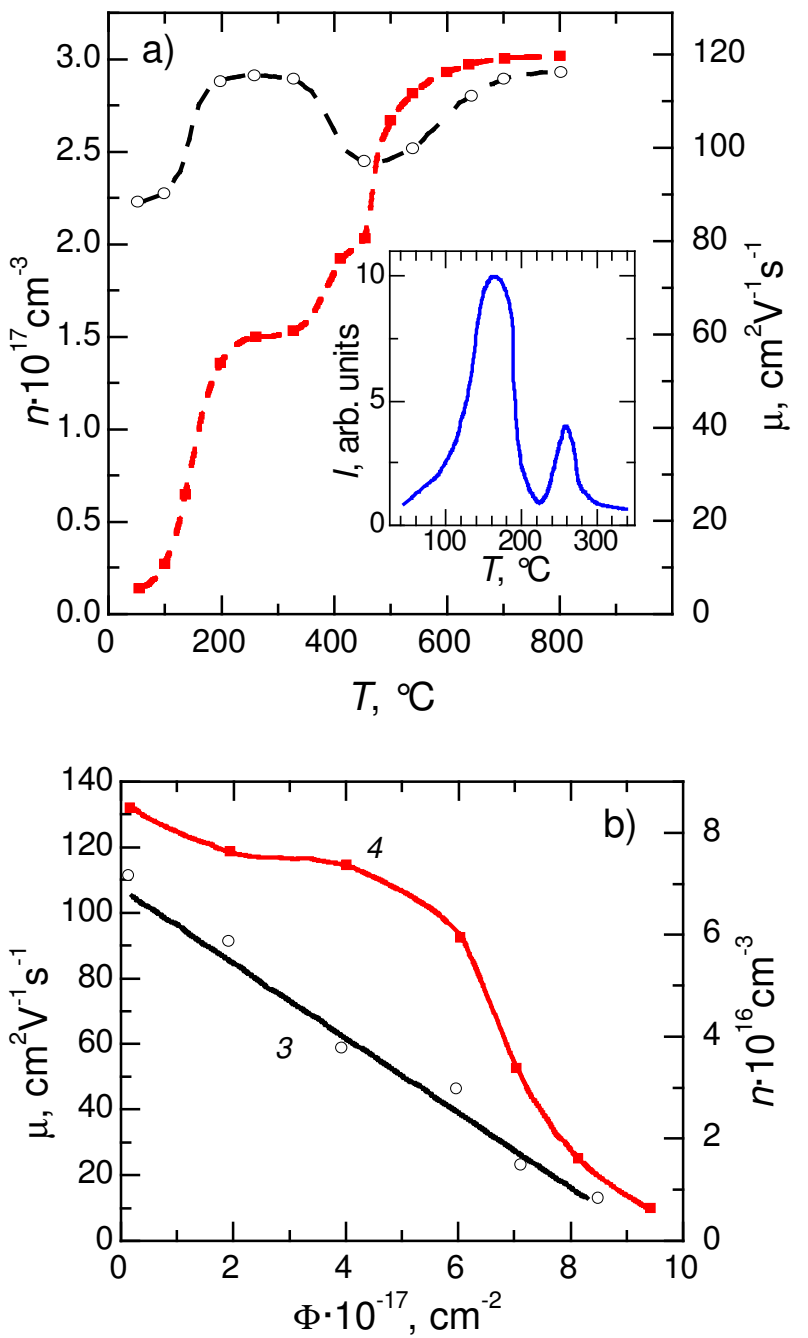

Fig. 3. Recovery of the electrical parameters of $\mathrm{GaP}$ crystal irradiated with $E=12 \mathrm{MeV}$ electrons, $\Phi=5 \cdot 10^{16} \mathrm{~cm}^{-2}$, in the process of isochronous annealing; 1 - concentration, $2-$ mobility (a). The electron concentration (3) and mobility (4) dependences on the fluence are given in (b). The curve 5 in the insert of (a) shows the conductivity relaxation amplitude as the annealing temperature function. 


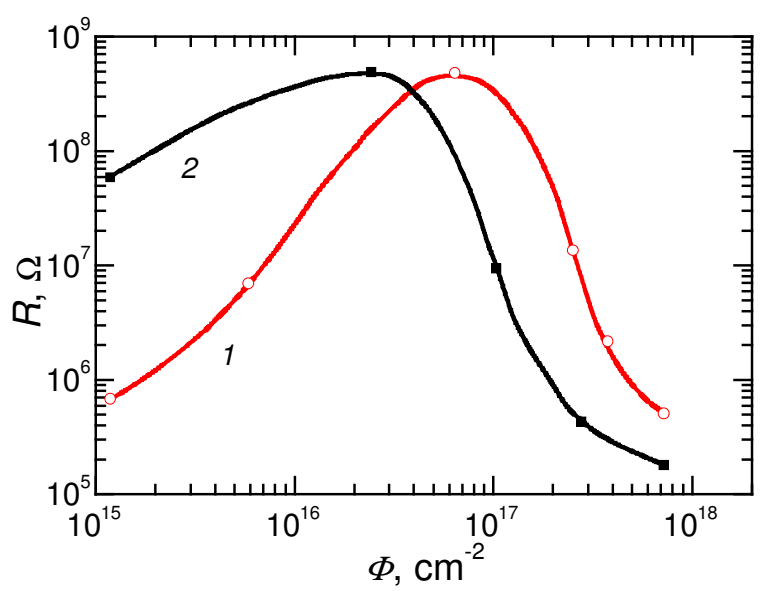

Fig. 4. Cadmium diphosphide resistivity dependence on the $14-\mathrm{MeV}$ electron fluence.

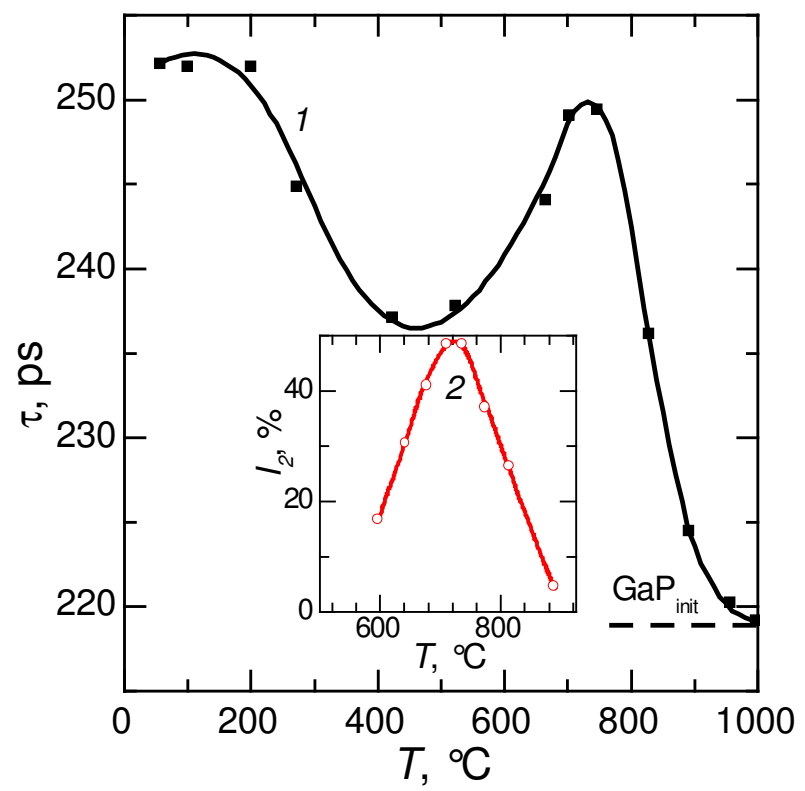

Fig. 5. The average positron lifetime dependence on isochronous annealing temperature. The long-living component curve is given in the insert.

One can see in Fig. 3 that the electron mobility annealing curve within $150 \ldots 350^{\circ} \mathrm{C}$ interval exhibits a wide maximum. Then, $\mu$ drops, and it might testify about transformation of the previously formed defects into those of a qualitatively new kind. One can strictly observe this transformation in the concentration recovery curve - specific contribution of the second annealing stage grows nearly to 50 percents (curve 1 ). The changes of the defect kind are supposed to be related with the increase of relaxation intensity (see curve 5).

It is possible to observe the transition of quantitative changes into the qualitative ones on kinetics of radiation defects accumulation, when enhancing the electron fluence. Fig. 4 shows resistivity dependence on the electron fluence for two cadmium diphosphide samples. The increase of defect concentrations causes the resistivity increase, but when $\Phi>10^{17} \mathrm{~cm}^{-2}$ another tendency is evident - resistivity drops monotonously and might be less than the initial one.
Measure of the positron lifetime during annealing in $\mathrm{GaP}$ irradiated with $80-\mathrm{MeV} \alpha$-particles under the fluence $10^{17} \mathrm{~cm}^{-2}$ demonstrates the same process of quantitative changes transition into the new qualitative ones (Fig. 5). The lifetime increase during irradiation is caused by the vacancy defect accumulation with a low electron density.

The decrease of positron lifetime testifies about vacancy annealing $\left(T<400{ }^{\circ} \mathrm{C}\right)$, but at $T>700{ }^{\circ} \mathrm{C}$ the lifetime grows due to formation of vacancy voids mainly possible from bivacancies or complex vacancy-type defects. We observe this phenomenon while analyzing the conductivity annealing curves of irradiated GaP (Figs 2 and 3$)$.

\section{Conclusion}

The irradiated crystal which as a non-equilibrium open system is able to demonstrate qualitative transition in the moment when a monotonously changing specific inner factor (which influence on system properties) reaches the critical value. It is clear from the above studied annealing process that it is the concentration of radiation defects that plays the role of this factor. When the defect concentration exceeds some critical value, the defects of new kind are formed: oscillation peaks in the isochronous annealing curve appear, and defects with a high crosssection of defect scattering and capture are created.

Accumulation of defects during irradiation might provoke an effect opposite to the initial one - the conductivity of the sample with high defect concentration has become greater than in the non-irradiated sample.

High-temperature annealing of the irradiated sample with the increased vacancy concentration causes appearance of the vacancy voids with the lower electron density.

It has been considered that the increase of defects concentration might be the reason for the qualitative changes in the electrical properties of irradiated crystals as well as increase in the intensity of diffusion processes during the high-temperature annealing.

\section{References}

1. Selishchev P.A. Self-organization in Radiation Physics. Kiev, Aspect-poligraf, 2004 (in Russian).

2. Sugakov V.J. The Basis of Synergetics. Kyiv, Oberehy, 2001 (in Ukraine).

3. Bianucci P., Mukherjee S., Hadi Tavakoli Dastjerdi M., Poole P., Mi Z. Self-organized InAs/InGaAsP quantum dot tube lasers. Appl. Phys. Lett. 2012. 101. 031104.

4. Litovchenko P.G., Makarenko V.G., Opilat V.Ya., Tartachnyk V.P., Tychyna I.I. Conductivity relaxation in irradiated gallium phosphide. Ukrainian Journal of Physics. 1988. 33, No 3. P. 387-390.

5. Radautsan S.I., Makarenko V.G., Opilat V.Ya., Tartachnyk V.P., Tychyna I.I. Conductivity relaxation and radiation defects' annealing in 
indium phosphide. Doklady Akademii Nauk USSR. Ser. A. 1988. 5. P. 50-53 (in Ukrainian).

6. Nemets O.F., Voyakov V.V., Litovchenko V.G., Makarenko V.G., Opilat V.Ya., Tartachnyk V.P., Tychyna I.I. Radiation defects in gallium phosphide. Doklady Akademii Nauk USSR. Ser. A. 1988. 5. P. 47-50 (in Ukrainian).

7. Konoreva O.V., Olikh Ya.M., Pinkovska M.B., Radkevych O.I., Tartachnyk V.P., Slapatska V.V. The influence of acoustic-dislocation interaction on intensity of the bound exciton recombination in initial and irradiated GaAsP LEDs structures. Superlattices and Microstructures. 2017. 102. P. 88-93.

\section{Authors and CV}

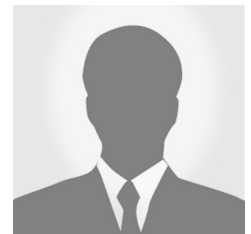

Nikita Zavada, born in 1984, postgraduate, Department of Radiation Physics, Institute for Nuclear Research, the National Academy of Sciences of Ukraine. Authored 2 publications. The area of his scientific interests includes research of radiation and growth defects in semiconductors and kinetics of defectimpurities complexes alteration.

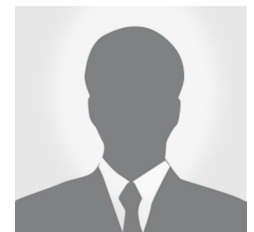

Oksana Konoreva, born in 1981, defended his Candidate Dissertation in Physics and Mathematics in 2012. Chief researcher, Department of Radiation Physics, Institute for Nuclear Research, the National Academy of Sciences of Ukraine. Authored over 45 publications. The area of his scientific interests includes research of radiation and growth defects in semiconductors and kinetics of defect-impurities complexes alteration.

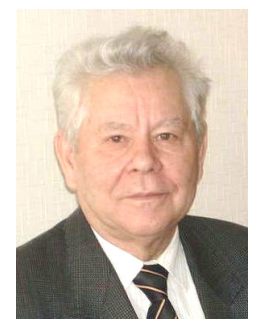

Piotr Lytovchenko, born in 1937, defended his Doctoral Dissertation in Physics and Mathematics in 1984 and became full professor in 1988. Senior researcher, Department of Radiation Physics, Institute for Nuclear Research, the National Academy of Sciences of Ukraine. Authored over 300 publications. The area of his scientific interests includes fundamental researches of the nuclear radiation effect on the physical properties of atomic and binary semiconductors and devices on their base.

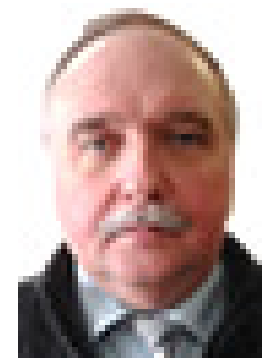

Vitaliy Opylat, born in 1959, defended his Candidate Dissertation in Physics and Mathematics in 1991. Associate Professor of Department of Nanophysics of Condensed Matter, Taras Shevchenko National University of Kyiv. Authored over 70 publications. The area of his scientific interests includes study of the nature of radiation defects in $\mathrm{A}^{3} \mathrm{~B}^{5}$ semiconductors and structures.

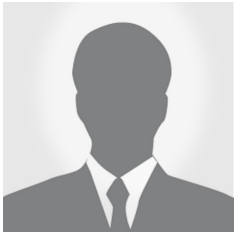

Miroslava Pinkovska, born in 1948. Chief researcher, Department of Radiation Physics, Institute for Nuclear Research, the National Academy of Sciences of Ukraine. Authored over 80 publications. The area of his scientific interests includes research of radiation and growth defects in semiconductors and kinetics of defect-impurities complexes alteration.

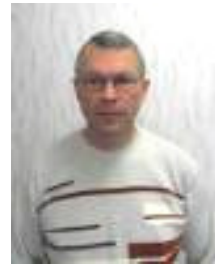

Olexander Radkevich, defended his Candidate Dissertation in Technical Sciences in 2010, Director of Research Institute of Microdevices, STC 'Institute for Single Cristals', the National Academy of Sciences of Ukraine. Authored over 20 publications, 2 patents. The area of his scientific interests includes creation of large integrated circuits and devices based on them.

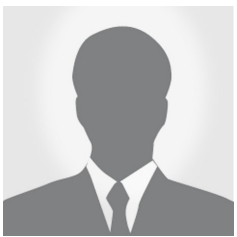

Vladimir Tartachnyk born in 1943, defended his Doctoral Dissertation in Physics and Mathematics in 1993 and became full professor in 2002. Senior researcher, Institute for Nuclear Research, the National Academy of Sciences of Ukraine, Department of Radiation Physics. Authored over 250 publications. The area of his scientific interests includes research of radiation and growth defects in semiconductors and kinetics of defectimpurities complexes alteration. 